\title{
The Effect of Kangaroo Mother Care Method toward Weight Gain and Length of Stay among Low Birth Weight Baby
}

\author{
Muliani, Lisnawati \\ Health Polytechnic in Palu, Department of Midwifery, Indonesia
}

\begin{tabular}{l} 
Article Info \\
\hline Article history: \\
Received Apr 11, 2018 \\
Revised Jun 5, 2018 \\
Accepted Jun 14, 2018 \\
\hline
\end{tabular}

\section{Keyword:}

Kangaroo Mother Care

Length of stay

Low birth weight

\begin{abstract}
Infant mortality needs to get serious attention. Special efforts are relatively easy and inexpensive in the handling and care is through treatment methods can improve the stability of Kangaroo Mother Care (KMC) for babies and breastfeeding. The effort hopefully will contribute to weight gain which take effect on the duration of treatment. The study design was Quasi Experiment with Prepost one group design. Samples were mothers with a history of low birth weight deliveries, with sample selection technique in consecutive sampling and sample number 36 babies. The samples criteria were infants birth weight between 1,000-2,100 gr, weight of infant when KMC was started between 900-2,100 gr, weight of infants post KMC were 1,300-2,500 gr, babies born with premature or small period of pregnancy. KMC method has the potential to improve the Weight on Low Birth Weight (LBW). Duration of KMC has no effect on weight gain but can accelerate the length of LBW in hospital. The longer do KMC, the shorter duration of hospital LBW in hospital. LBW should be treated KMC to accelerate weight gain and reducing the length of hospitalization.
\end{abstract}

Copyright $(0) 2018$ Institute of Advanced Engineering and Science. All rights reserved.

\section{Corresponding Author:}

Muliani,

Health Polytechnic in Palu,

Department of Midwifery, Indonesia.

Email: murid_lewa@yahoo.co.id

\section{INTRODUCTION}

First year of life is the most important period as a time of growth and development of children will go with the immense influence on the formation of the quality of life of children in order to be able to act and function optimally. It is necessary adjustments to life as a process of transition is a critical phase for the baby's life from intrauterine to extrauterine.

Birth of LBW infants, especially premature births entire organ in the body is not functioning optimally, including sucking and swallowing reflexes are still weak and very dependent on gestational age. Lack of body fat reserves and system of regulation of body temperature in infants immature to make a baby should be treated incubator/box with lights warmers, so the method KMC through the arms on the mother's chest in direct contact skin of the mother with the baby's skin is considered effective enough to help the baby stay warm, so can prevent energy loss due to excessive loss of body heat. This thus potentially increase the nutritional intake (breast milk) as well as help increase weight impacting on increasingly shortened treatment period.

The method of Kangaroo Mother Care (KMC) is very usefull: 1) the infant can return home from the hospital early; 2) succesfulnees of exclusive breastfeeding. KMC is also as basic interventions in the nursing care unit of the Neonatal Intensive Care Unit (NICU) the child is to support self-confidence and parental competence, educate and provide services to enhance the bonds of babies optimally [1].

Based on the report by the World Health Organization (WHO) estimated that over 20 million babies born are low birth weight (LBW) as much as $15.5 \%$ of all births and $95 \%$ in developing countries, while for 
Asian countries reached 18.3 LBW births $\%$ of the 77,490 babies born alive as well as 4 million neonatal deaths $28 \%$ were preterm birth. Similarly, preterm infant mortality is still high in developing countries such as Indonesia and according to the world that preterm births reached $75-80 \%$ of all babies who died at the age of less than 28 days [2].

Based on the report of WHO (2015a) for a period of two decades that the live birth rate increased from the year 2000 amounted to 127.7 million to 137.7 million in 2013. Of that number there were 6.3 million deaths of children $<5$ years $(46 / 1,000$ live births) and $44 \%$ of them are in neonatal mortality. The main cause of death was complications of premature birth by $17 \%$ of all deaths, and 21/1,000 live births occur in developing countries, particularly in Southeast Asia region reached 13/1,000 live births [3].

Neonatal mortality rate is predicted to increase from $45 \%$ in 2015 to $52 \%$ in 2030 , therefore there are 63 countries which should accelerate the process of achieving the target SDGs that is 12/1,000 live births in 2030 [4]. Nationally Central Sulawesi increased Infant Mortality Rate (IMR) from 52 to 60 per 1,000 live births, so that IMR ranks third highest after West Sulawesi (74/1,000 live births) and Nusa Tenggara Barat (72/1,000 live births). Infant Mortality Rate programmatically problem in Central Sulawesi need to get serious attention because it has increased about $15 \%$ over the last 5 years. The still high IMR as an indication that the problem of health and general development in Central Sulawesi province requires a special effort. Cause highest IMR is the birth of low birth weight and asphyxia) [5]. Based on data from the Health Office of Central Sulawesi Province in 2013 live birth rate reached 49,755 and IMR as many as 113 babies being born low birth weight and 94 babies died by asphyxia, 2014 increased by live births 49.756, infant mortality for LBW 117 infants and death due to asphyxia 116 baby [6].

Similarly, the birth rate in hospital Anutapura Palu in 2013 the number of live births, 3.848 and 223 such births, 2014 to increase the number of live births, 4.060 and 264 diantranya was the birth of low birth weight, but the implementation of this method has not been applied in hospitals optimally and forms of socialization has not been fully received a positive response from the mothers, so it tends to reject the application of the method, because of the lack of standard operating procedures that support the implementation of the KMC method) [7].

Results of the study are randomized controlled trial (RCT) between the KMC method is direct skin contact with the baby's mother with conventional methods incubator to the physical stability at 1,200-2,199 grams birth weight infants, indicating that skin contact with the skin in preterm infants is an alternative the first well in life after birth [8]. Several studies show that treatment with KMC method provides a positive effect on breastfeeding (breast milk) and baby's growth compared to treatment with an incubator as in research Hurst that the increased volume of milk per day in 4 weeks and exclusive breast respectively of 647 $\mathrm{ml}$ and $37 \%$ in the KMC group was $530 \mathrm{ml}$ and $6 \%$ in the control group. Similarly, the RCT study by Charpak or partially exclusive breastfeeding for 3 months up to $82 \%$ in the KMC group and $75 \%$ in the control group, and Cattaneo in WHO shows exclusive breastfeeding after discharge $88 \%$ in the KMC group and $70 \%$ in the control group [9].

Research Systematic reviews indicate that treat the mother's skin contact with baby's skin is very effective in preventing hypothermia in preterm infants or low birth weight and the Relative Risk (RR) 0.09; 95\%; CI 0.01 to 0.64; Number Needed to Treat (NNT) 2; 2-4 means that every second LBW infants who were treated with the methods of KMC will prevent the baby on the incidence of hypothermia [10] and regularly increasing body weight per day until discharge [11].

One advantage of the methods of KMC is the effect on the baby's growth as the result of research that infants who were treated with the methods of KMC significantly grow better by the end of the study, in which a small baby with KMC occurred significantly greater improvement on Weight, body length, head sircumference $(2,388$ grams, $47.8 \mathrm{~cm}, 33.4 \mathrm{~cm})$ than from the baby with the conventional method $(2,065$ grams, $46.4 \mathrm{~cm}, 32.1 \mathrm{~cm})$ at the end of the monitoring with $\mathrm{p}<0.05$. Another positive effect shortening the period of hospitalization with an average (mean \pm SD) at KMC CMC 12.78 \pm 6.72 and 12.86 \pm 5.77 $(\mathrm{p}=0.93)$. KMC method can also extend the duration of breastfeeding, stabilizing milk production, increase the amount of nutrients each day, and enhance competence in breastfeeding mothers [12].

The results of another study of 50 infants (weight $<2,000 \mathrm{~g}$ ) at 28-32 weeks gestation, who received the KMC method for at least 4-6 hours/day showed an increase in weight (grams) (mean \pm SD) 29 \pm 3.52 ; Hospital treatment duration (mean \pm SD) of $15.5 \pm 11.3$ days; age at discharge (mean \pm SD) 23.6 \pm 3.52 days, while 20 of them infants who were followed for 8 weeks after discharge significantly increased weight $1.135 \pm 0.121$ and only mothers who do KMC method at home after going out of RS [13].

Likewise, the results of research on that significantly there are significant differences between the mean difference of BB baby at home until the next re-visit during the three visits that have been categorized in the duration of $\mathrm{KMC} \geq 4 \mathrm{jam} /$ day with each increment of the average increase in body weight $(80.7$ grams; $297.5 \mathrm{~g} ; 400.4$ grams) with a confidence interval (CI) 95\% (from 57.6 to 103.8; 241.4 to $353.5 ; 297.8$ to 502.9) and each p-value 0.001, with the results of the analysis (X2) on repeat visits I-III, that the KMC babies 
$\geq 4$ hours/day have a positive effect $\geq 30$ gram baby's weight gain/day for 2 times great to visit the second anniversary of the KMC infants $<4$ hours / day. In the first and second visit there significance in clinics and with less statistical p-value $<0.05$ [14].

\section{RESEARCH METHOD}

This research is an analytic study design Quasi Experiment with Prepost approach one group design. The target population in this study are all mother-infant pairs with LBW births and samples are all over the mothers of infants with low birth weight birth history both mature and prematurely treated at Hospital Perinatal Lounge Anutapura Palu and willing to carry out the treatment with the KMC method. The total sample of 36 infants, were selected by consecutive sampling. The confounding variables was controlling using selected criteria with infants birth weight between 1,000-2,100 gr, weight of infant when KMC was started between 900-2,100 gr, weight of infants post KMC were 1,300-2,500 gr, babies born with premature or small period of pregnancy.

\section{RESULTS AND ANALYSIS}

Of whole KMC babies do care the average baby increases exceeding BB Gold Standard of the World Health Organization (BB increase in average $\geq 15 \mathrm{~g} /$ day. The results can be seen in the univariate and bivariate analysis in the form of paired samples test on a group of data pairs with normality test beforehand, and Independent Samples test group unpaired data.

Table 1 shows that the birth of a baby with low birth weight categories and each LBW infants 18 infants $(50.0 \%)$, infants who gain weight as much as 21 infants $(58.3 \%)$ and 15 infants $(41.7 \%)$ were not increased weight.

Table 1. Characteristics of Infants Who Receive Treatment Methods KMC

\begin{tabular}{llcc}
\hline & Characteristics baby & $\begin{array}{c}\text { Frequency } \\
(\mathrm{n}=36)\end{array}$ & $\begin{array}{c}\text { Percentage } \\
(\%)\end{array}$ \\
\hline \multirow{2}{*}{ Birthweight } & $1,000-1,500$ gram & 18 & 50.0 \\
\multirow{2}{*}{ Weight before KMC } & $1.501-2.100$ gram & 18 & 50.0 \\
\multirow{2}{*}{ Weight after KMC } & $900-1,500$ gram & 20 & 55.6 \\
\multirow{2}{*}{ Weight changes } & $1,501-2,100$ gram & 16 & 44.4 \\
\multirow{2}{*}{ LBW by pregnancy } & $1,300-1,500$ gram & 9 & 25.0 \\
& $1,501-2,100$ gram & 27 & 75.0 \\
& Increase & 21 & 58.3 \\
& Not increased & 15 & 41.7 \\
& Small for gestational age & 7 & 19.4 \\
\hline
\end{tabular}

Paired samples t-test was done to test the results of the implementation of the KMC method of weight gain in infants before and after the KMC method. Prior to the analysis of paired samples t-test was first tested nommalitas and showed normal distribution of data with a p-value> 0.05, for weight before KMC 0.335 and body weight after KMC 0.050 based on test Shapiro-Wilk according to criteria of the number of samples is less than 50 baby.

To see whether there is any potential for KMC method to increase length of stay for KMC hospital, and determine the average weight difference before $\mathrm{KMC}$ to assess the weight at home as well as the increase in the average weight/day. Table 2 shows that there is an increase in body weight was significantly based on the test results of pre-post weight before and after the KMC to gain weight 188.75 grams and a standard deviation of 166.6 and indigo p-value 0.000 . KMC method has the potential to increase weight gain in LBW.

Table 2. Paired Samples Test with a mean difference Improvement Weight Loss Before and After KMC on LBW

\begin{tabular}{|c|c|c|c|c|c|c|}
\hline KMC Method & $\mathrm{N}$ & Mean \pm SD & $\Delta$ & CI $95 \%$ & t-test count & $p$-value \\
\hline Weight before KMC & 36 & $1,555 \pm 327.08$ & $188.75 \pm 166.66$ & $132.4-245.1$ & 6.795 & 0.000 \\
\hline Weight after KMC & 36 & $1,743 \pm 236.03$ & & & & \\
\hline
\end{tabular}

In this study also shows the results of an analysis of potential methods of KMC on LBW to the length of using Independent analysis of test samples as in the Table 3. This table informs that the long day on 
the duration of hospitalization KMC on LBW infants each with a duration <4 hours/day own (Mean \pm SD) $8: 46 \pm 7: 15$ today and $\geq 4$ hours/day $6: 50 \pm 4: 24$ today with p-value $=0133$, the results of the analysis showed no statistical significance.

Table 3. Independent Sample Test on Day Care Old KMC with Duration of KMC in LBW

\begin{tabular}{llcllll}
\hline & Duration of KMC & $\mathrm{N}$ & Mean \pm SD & CI 95\% & t-test count & $p$-value \\
\hline \multirow{2}{*}{ Long day care KMC } & $<4$ hours / day & 28 & $8.50 \pm 7.15$ & $7.39-3.46$ & 0.736 & 0.133 \\
\multirow{2}{*}{ Difference in weight gain } & $\geq 4$ hours / day & 8 & $6.50 \pm 4.24$ & & & \\
& $<4$ hours / day & 28 & $1.99 \pm 180.45$ & $181.87-91.87$ & 0.668 \\
& $\geq 4$ hours / day & 8 & $1.54 \pm 107.16$ & & & \\
\hline
\end{tabular}

Similarly, the duration of KMC to weight gain by the difference in weight gain is relatively not experienced significant difference. It is proved that the duration of $<4$ hours/day had an average weight gain (mean \pm SD) $1.99 \pm 180.45 \mathrm{~g}$ and $\geq 4$ hours/day with an average weight gain 1:54 \pm 107.16 and $\mathrm{p}$-value 0.187 .

\section{DISCUSSION}

Kangaroo Mother Care as one simple treatment at LBW in fostering better health by improving the effectiveness of the control body temperature and bonding baby's mother, exclusive breastfeeding, to prevent infection. Caring for a baby with KMC method continuously by direct contact with the skin and helps skin completely exclusive breastfeeding. KMC method can be started in hospital and continued at home.This research is trying to integrate with the view to increase the potential of KMC method weight and length of treatment at the time LBW KMC method for care in hospital.

\subsection{The Potential Application of the KMC Method to Increase BB Low Birth Weight (LBW)}

Overall the study subjects were 36 infants. The results of the analysis of normality test, normal distribution of data so that it can proceed with the analysis Paired sample test by measuring the weight prior to methods of KMC and continued with the implementation of KMC every day for at least 2 hours/periods, and weigh the baby every day in the morning until baby otherwise meet the criteria of return. Next calculate the average weight gain of the babies during treatment methods KMC.

The test results show that babies gain weight during treatment methods KMC average of $188.75 \pm 166.6$ grams with a p-value of 0.000 in the range of 132.4-245.1 CI 95\%. The result means that statistically significantly KMC method application has the potential to increase weight on LBW.

KMC as an intervention method simple and easy to implement and readily accepted by most mothers during hospitalization which provides many benefits and reduce the risk of hypothermia without side effects. It also gives important implications in the treatment of low birth weight in developing countries where conventional treatment with expensive facilities are not available in all places [15]. Baby care with KMC method can be introduced during the mother in the hospital for further applied at home until the baby is urged to come out or feel uncomfortable anymore which usually took 40-41 weeks of age corrected. Some studies do methods of KMC at home with the shortest period of 2-4 hours/day showed the baby's condition is stable [16].

It can be concluded that the baby's birth LBW will have a positive effect if done treatment methods KMC since in hospital until the baby home and continued at home is minimal with the shortest period of 2 hours/day and its duration can be increased gradually to maintain stability of the baby's condition and weight gain adequate body. KMC method implementation can be done without disrupting the daily activities of the mother, but require habituation and perseverance mother and family support. It is also supported by a study by the KMC method also increases the weight of the baby after discharge compared to the control group by 3.6 times, CI $95 \%$ in the interval from 0.8 to 6.4 clinically meaningful [12]. Implementation of the KMC method in the UK 28-32 weeks, at least 4-6 hours/day showed a significantly increased weight $1.135 \pm 0.121$ for mothers who perform the method KMC at home [13]. Results of other studies with direct skin contact of mother and baby show an increase in the average weight of 30 grams/day [17].

\subsection{The Duration of KMC Method to Long Day Care Infant Low Birth Weight (LBW)}

The results of the analysis in Table 3 shows that the old days of the duration of treatment KMC on LBW infants, were not statistically significant with p-value 0.133. Similarly, the duration of KMC to weight gain by the difference in weight gain was relatively significant differences, it is proved that the duration $<4$ hours/day had an average weight gain (mean \pm SD) $1.99 \pm 180.45 \mathrm{~g}$ and $\geq 4$ hours/day with an average weight gain 1:54 \pm 107.16 and p-value 0.187 .

IJPHS Vol. 7, No. 2, June 2018: 91 - 96 
Thus the duration of KMC $<4$ hours/day are more likely to gain weight than of $\geq 4$ hours/day, but the superiority duration of $\mathrm{KMC} \geq 4$ hours/day contained in the length of stay of 6.5 days shorter than the duration $<4$ hours/day the length of stay to 8.5 days. This means that the longer the duration of a baby in KMC greater the opportunity to shorten the length of stay, which will further lower the cost of care. The average treatment duration of direct contact of skin to skin on the baby group KMC showed a tendency length of stay in hospital is shorter (8.6 days) compared with Conventional Method Care (CMC) with length of stay (9.3 days) [15].

Similarly, other studies show the method KMC, as a means of heating and can shorten the length of stay in hospital, so it does not require a large fee also showed weight gain and decrease the incidence of hypothermia compared to infants who did not receive the method of KMC [18]. In line with research in Bogota that the use of KMC dominant show more effective results against weight gain were $13.11 \pm 10.04 \mathrm{gm}$ at KMC group compared with $15.81 \pm 3.33 \mathrm{gm}$ in the control group by value (p-value $<0.001$ ). Similarly Ruiz in 2016, the incidence of hypothermia $(14.6 \%)$ in the control group and $(5.1 \%)$ in the group of KMC ( $\mathrm{p}$-value $=0048)$, duration of hospital stay is shorter $(\mathrm{p}$-value $=0.015)$ as well as savings.

The research results prove that the implementation of the KMC method have an increasing impact on LBW $\mathrm{BB} \geq 30 \mathrm{~g} /$ day with a duration $\geq 4$ hours/day for at least 2 hours / periods KMC, potentially amounting to 3.5 times greater than the KMC <4 hours/day, CI 95\% (1.2 to 9.8). Some babies feel comfortable with KMC method so the weight is $>2,500 \mathrm{~g}$ with an upright body position [14]. Other studies have shown that some mothers feel sad, guilty, scared, anxious, insecure and hesitant when recommended start KMC, but after carrying out the method KMC mothers find more positive effects of direct skin contact, feel the love and affection of touch, breastfeeding, and women are more confident in caring for her baby. Fiveteen women who participated in this study of 14 women are more confident after carrying out the method KMC and to 14 women will continue KMC method at home and seven of them carrying out the method KMC mothers after discharge up to >4 weeks [19].

\section{CONCLUSION}

The results of this study can be concluded that there is a potential method of Kangaroo Mother Care (KMC) to the increased weight and length of the Infant Low Birth Weight (LBW) in Palu Anutapura Hospital. To improve the stability condition of LBW infants and accelerate weight gain and reduce the duration of hospital baby in a hospital, it can be suggested that the hospital or policy-makers to run the Standard Operating Procedure (SOP) for each baby with a history of low birth weight births.

\section{REFERENCES}

[1] Johnson, AN., "Kangaroo Holding beyond the NICU", Journal of Pediatric Nursing, 31(1); 53-56, 2005.

[2] WHO, "Low Birthweight: Country, Regional and Global Estimates", Unicef, New York, 2004.

[3] WHO, "World Health Statistics 2015", Luxembourg, 2015a.

[4] WHO, "Levels \& Trends in Child Mortality, Report 2015", Unicef, New York, 2015b.

[5] Data and Information Center Ministry of Health RI (PUSDATIN Kemenkes RI), "Executive Summary, Health Data and Information of Central Sulawesi Province (in Bahasa)", 2013.

[6] Public Health Office of Mid Sulawesi Province, "Health profile of Mid Sulawesi Province", UPT Survailans, Data and Information, 2015.

[7] Anutapura Palu Hospital, "Report of labor and infant birth in 2013-2014", 2015.

[8] Kennel JH., "Randomized controlled trial of skin-to-skin contact from birth versus convenstional incubator for physiological stabilization in 1200 to 2199 gram newborn", Acta Pediatrica 95: 15-16, 2006.

[9] WHO, "Kangaroo Mother Care: a Practical Guide", Geneva, 2003.

[10] McCall EM., Alderdice FA., Halliday HL., Jenkins JG., Vohra S., "Interventions to Prevent hypothermia at birth in preterm and/or LBW infant (review)", The Cochrane Collaboration Sys Rev: 1-15, 2007.

[11] Conde-Agudelo, Diaz-Rosello JL, \& Belizan JM., "Kangaroo Mother Care to Reduce Morbidity and Mortality in Low Birth Weight Infants", Cochrane Database Syst Rev, 2003;(2):CD002771, 2003.

[12] Thukral, A., Chawla, D., Agrwal, R., Deorari AK. \& Paul, VK., "Kangaroo Mother Care an Alternative to Conventional Care", All India Institute of Medical Sciences (AIIMS), NICU Protocols, Division of Neonatology Departement of Pediatrics, New Delhi, 1-15, 2008.

[13] Gupta, M., Jora, R., Bhatia, R., "Kangaroo Mother Care (KMC) in LBW Infants-a Western Rajasthan Experience", Indian Journal Pediatric, 74(8):747-49, 2007.

[14] Muliani, Ismail Dj., \& Haksari LE., "Effect of kangaroo mother care (KMC) duration at home on weight gain in low birth weight infant (LBW) during control”, Jurnal Ilmiah Ilmu Kesehatan Poltekkita, Vol.2 Pp. 545-560, Palu, October 2012, ISSN 1907-459X, 2012.

[15] Kadam S., Binoy S., Kanbur W., Mondkar J.A. and Fernandez A., "Feasibility of Kangaroo Mother Care in Mumbai”, Indian Journal of Pediatrics, Januari 2005, Vol. 72 (1): 35-38, 2005. 
[16] Blackwell, K. \& Cattaneo, A., "What is the evidence for kangaroo mother care of the very low birth weight baby?", International child health review collaboration; 1-3, 2007.

[17] Subedi, K., Aryal, DR., Gurubacharya, SM., "Kangaroo Mother Care for Low Birth Weight Babies: a prospective observational study”. Paropakar Maternity and Women's Hospital, Thapathali, Kathmandu, Nepal. J. Nepal Pediatric. Soc. 29(1); 6:9, 2008.

[18] Canodia R., Bora R., Gupta A., "Kangaroo Mother Care: a cost efektive and an alternate method to manage hypothermia in low birth weight babies for better clinical outcome". Value in Health 19 (2016) A347-A766, 2016.

[19] Endyarni, B., Roeslani, RD., Rohsiswatmo, R. \& Soedjatmiko, "Mother's response on Kangaroo Mother Care intervention for preterm infants", Departement of child health, medical school, University of Indonesia, Cipto Mangunkusumo Hospital, Jakarta. Paediatrica Indonesia, 49(7); 224-28, 2009. 